\title{
Assessing Reliability by Multidimensional Convolution with Quantization
}

\author{
Becker, Peter W.; Jarkler, Björn
}

Published in:

I E E E Transactions on Reliability

Link to article, DOI:

10.1109/TR.1974.5215247

Publication date:

1974

Document Version

Publisher's PDF, also known as Version of record

Link back to DTU Orbit

Citation (APA):

Becker, P. W., \& Jarkler, B. (1974). Assessing Reliability by Multidimensional Convolution with Quantization. I E E E Transactions on Reliability, R-23(3), 214-218. https://doi.org/10.1109/TR.1974.5215247

\section{General rights}

Copyright and moral rights for the publications made accessible in the public portal are retained by the authors and/or other copyright owners and it is a condition of accessing publications that users recognise and abide by the legal requirements associated with these rights.

- Users may download and print one copy of any publication from the public portal for the purpose of private study or research.

- You may not further distribute the material or use it for any profit-making activity or commercial gain

- You may freely distribute the URL identifying the publication in the public portal

If you believe that this document breaches copyright please contact us providing details, and we will remove access to the work immediately and investigate your claim. 


\title{
Assessing Reliability by Multidimensional Convolution with Quantization
}

\author{
PETER W. BECKER, ASSOCIATE MEMBER, IEEE, AND BJÖRN JARKLER
}

\begin{abstract}
Consider a system with several input parameters; each input is a stochastic variable. The joint probability of all system outputs simultaneously meeting their specifications is found by a hitherto unpublished method called "Multi-dimensional Convolution with Quantization." To use the method, two assumptions must be satisfied; the assumptions are milder than the two popular assumptions of linearity and statistical independence. There is one example.
\end{abstract}

READER AIDS:

Purpose: Widen state of the art

Special math needed for explanations: None

Special math needed for results: None

Results useful to: Reliability engineers

\section{INTRODUCTION}

In this paper a new and simple method is presented called "Multidimensional Convolution with Quantization" (MDCQ) by which the following well-known problem can be solved [1]. Consider a system or circuit with specified topology, where each of the input parameters has a nominal value and a true value, and where the pdf of the true value of each of the $n$ input parameters is known. The system is said to meet the output specifications when and only when the true value of each output parameter satisfies a specified constraint; the problem at hand may now be stated: with what probability, $\mathscr{R}$, will the $m$ system outputs simultaneously satisfy the $m$ output constraints? The probability $\mathscr{R}$ is the reliability of the system. If we are only concerned with the system at production time, the probability is called the production yield.

\section{Notation}

$x_{i}$ true value of input parameter $i$

$x_{i, \text { nom }}$ nominal value of input parameter $i$

$f_{i}\left(x_{i}\right)$ pdf $\left\{x_{i}\right\}$

$y_{j}$ true value of output-parameter $j$

$z_{j}$ transformed $y_{j}$

$n$ number of input-parameters, $i=1, \ldots, n$

$m$ number of output-parameters, $j=1, \ldots, m$

min, max subscripts which imply the minimum or maximum allowed value of an output parameter

$\mathscr{R}$ system reliability (probability that all $m y_{j}$ simultaneously satisfy the output constraints

$\Phi_{i} \equiv x_{i} / x_{i, \text { nom }}$

$O_{i}$ transformed $\Phi_{i}$

$f$ joint pdf of the $x_{i}$

$h_{j} \quad y_{j}=h_{j}\left(\Phi_{1}, \ldots, \Phi_{n}\right)$

$g$ joint pdf of the $y_{j}$

\section{THREE INHERENT DIFFICULTIES}

To simplify the following discussion we will use normalized true values, $\Phi_{i}$, for the input variables. The $n$ nominal values are constants in the ensuing material and, therefore, will be omitted. The true value of $y_{j}$ is a function of the $n$ values of $\Phi_{i}$ :

$$
y_{j}=h_{j}\left(\Phi_{1}, \ldots, \Phi_{n}\right)
$$

The problem we are concerned with is complicated for three reasons.

(1) The $\Phi_{i}$ will almost always show some form of statistical dependence. This means that the joint $\operatorname{pdf} f=f\left(\Phi_{1}, \ldots, \Phi_{n}\right)$ is probably not a product of $n$ pdf's, $f_{i}\left(\Phi_{i}\right)$. This is an exasperating fact of life as the designer in general has information only about the $f_{i}\left(\Phi_{i}\right)$.

(2) The $y_{j}$ are usually nonlinear functions of the $\Phi_{i}$.

(3) The true values of the $y_{j}$ are (almost always) statistically dependent for the following two reasons. (a) They are all functions (though different functions) of the same set of $\Phi_{i}$. (b) Each $y_{j}$ reflects to what degree that particular system has a certain property; as these properties often are related (e.g. gain and bandwidth) one expects a statistical relationship among the $y_{j}$.

\section{TWO WAYS TO DETERMINE THE RELIABILITY}

When one attempts to find $\mathscr{R}$, there are essentially two different approaches. 1) estimate $\mathscr{R}$ directly; this is the road one follows if one uses Monte Carlo techniques [2]. 2) solve what appears to be a more difficult problem, namely, determine $g=g\left(y_{1}, \ldots, y_{m}\right)$, the joint pdf for the $m$ output variables. When $g$ has been established it is a simple matter to determine $\mathscr{R}$.

$$
\mathscr{R}=\int_{y_{1, \min }}^{y_{1, \max }} \ldots \int_{y_{m, \min }}^{y_{m, \max }} g \cdot d y_{m} \ldots d y_{1}
$$

A method where one determines $\mathscr{R}$ by way of $g$ is the socalled "Normal approximation method" [3]. With the MDCQ-method we likewise determine $g$ before $\mathscr{R}$ is computed.

\section{TWO ASSUMPTIONS}

If one knows $f$ and all $h_{j}$, one can in principle estimate $g$ by simulating a large number of representative systems and then determine $\left(y_{1}, \ldots, y_{m}\right)$ for each system; the $m$ dimensional histogram would then constitute an estimate of $g$. In practical cases this approach is out of the question as 
the computational work would be horrendous. The way to arrive at an expression for $g$ with less work is to introduce some simplifying yet realistic assumptions. We will now introduce two such assumptions. When both assumptions apply the MDCQ-method is applicable. In Section 5 we will, by example, demonstrate how easily $g$ and $\mathscr{R}$ can be obtained by the MDCQ-method.

\section{Assumption 1}

Each $h_{j}$ is essentially a sum of functions each of which depends on only one input variable. E.g., for $n=4, h_{j}$ could have the decomposed form of the sum of four 1argument functions as in (3); this is the most desirable state of affairs. If, however, $h_{j}$ takes the form of two 1-argument functions added to one 2-argument function, as illustrated in (4), we can also live with that situation.

$$
\begin{aligned}
& y_{j}=h_{j, 1}\left(\Phi_{1}\right)+h_{j, 2}\left(\Phi_{2}\right)+h_{j, 3}\left(\Phi_{3}\right)+h_{j, 4}\left(\Phi_{4}\right) \\
& y_{j}=h_{j, 1}\left(\Phi_{1}\right)+h_{j, 23}\left(\Phi_{2}, \Phi_{3}\right)+h_{j, 4}\left(\Phi_{4}\right)
\end{aligned}
$$

In practical cases the range of $\Phi_{2}$ and $\Phi_{3}$ can be represented by a few, say five, typical values. If so $\left(\Phi_{2}, \Phi_{3}\right)$ in (4) can be regarded as a new discrete variable $\Phi_{23}$ with " $5 \times 5=$ 25 " values. When $\left(\Phi_{2}, \Phi_{3}\right)$ is substituted for by $\Phi_{23}$ in all equations like (4) we have substituted a 2-argument function by a 1-argument function and reduced the number of input values from $n$ to $(n-1)$. At the same time we now must compute 25 values of the $\Phi_{23}$-functions rather than the 5 values of the $\Phi_{2}$-functions plus the 5 values of the $\Phi_{3}$-functions which would have sufficed had the $h_{j, 23}$-function been the sum of a $h_{j, 2}$-function and a $h_{j, 3}$-function in all $m$ equations. This is the "essentially additive" assumption. It is much less restrictive than the assumption of linearity which is made so freely in the literature, e.g., in connection with sensitivity and coefficient-of-variation-analysis [4]. A suitable transformation sometimes can be used to decompose $h_{j}\left(\Phi_{1}, \ldots, \Phi_{2}\right)$; the example in Section 5 illustrates this point.

\section{Assumption 2}

The $n$-variate pdf $f\left(\Phi_{1}, \ldots, \Phi_{n}\right)$ is essentially the product of the marginals, i.e., the $n$ input variables are statistically independent. E.g. for $n=4, f$ could have the decomposed form of (5), which is the most desirable state of affairs; if, however, $f$ takes the form shown in (6) we can also live with that situation.

$$
\begin{aligned}
& f\left(\Phi_{1}, \ldots, \Phi_{4}\right)=f_{1}\left(\Phi_{1}\right) \cdot f_{2}\left(\Phi_{2}\right) \cdot f_{3}\left(\Phi_{3}\right) \cdot f_{4}\left(\Phi_{4}\right) \\
& f\left(\Phi_{1}, \ldots, \Phi_{4}\right)=f_{12}\left(\Phi_{1}, \Phi_{2}\right) \cdot f_{3}\left(\Phi_{3}\right) \cdot f_{4}\left(\Phi_{4}\right) .
\end{aligned}
$$

We would like to substitute the bivariate $\operatorname{pdf} f_{12}\left(\Phi_{1}, \Phi_{2}\right)$ in (6) with a univariate pdf. Again our procedure will be to use just a few, say five, representative $\Phi_{1}$-values and $\Phi_{2}$-values. We then regard $\left(\Phi_{1}, \Phi_{2}\right)$ in each $h_{j}$ as a new discrete variable $\Phi_{12}$ which can have " $5 \times 5=25$ " possible values. If it so happens that $\Phi_{1}$ and $\Phi_{2}$ are the arguments for a 2-argument function, like $h_{j, 23}$ in (4). we are in luck ; then $\Phi_{1}$ and $\Phi_{2}$ are already regarded as a new variable $\Phi_{12}$ with 25 values. If the situation is as illustrated by (4) and (6), it means that to remove the bivariate $\operatorname{pdf} f_{12}$ we have to regard $\left(\Phi_{1}, \Phi_{2}, \Phi_{3}\right)$ as a new discrete variable $\Phi_{123}$ which can take " $5 \times 5 \times 5=$ 125 " different values, each of which must be treated separately when we compute the $m$-variate $g$. When $\Phi_{123}$ must be used instead of just treating $\Phi_{1}, \Phi_{2}$ and $\Phi_{3}$ separately (as done when (3) and (5) are true) the number of $y_{j}$-values and associated probabilities which must be computed separately increases sharply from $(5+5+5)$ to $(5 \times 5 \times 5)$. Only when the 125 values of $y_{j}$ and the associated probabilities are known, can we convolve the $(n-3)$ pdf's in the manner to be described shortly. The assumption of the $n$ input variables having "essentially statistical independence" is not as restrictive as it may sound for the following reason. In practice it rarely is possible to make all the measurements necessary to determine $f$; so only the $n$ marginals are known. The designer must decide upon an $f$-function to compute the yield; therefore, as long as he has no information to discredit the assumption, he might just as well assume that the $n$ input variables are stastistically independent. When the MDCQ method is used, the $n$ marginal pdf's can have different and arbitrary forms as long as the range of each input variable is finite; they are not limited to Normality as done so often in the literature.

\section{EXAMPLE: THE YIELD OF A $R C L$-CIRCUIT}

\subsection{Some Features of the RCL-Circuit}

We want to illustrate how multidimensional convolution can be used to assess the performance of an ensemble of circuits built to the same set of specifications. Especially we want to compute the reliability $\mathscr{R}$. To that end we have selected a simple circuit, the series combination of a capacitor, inductance, and resistor with true values $C, L$ and $R$. The resistor can represent the loss in a realizable inductance. The circuit has the characteristic equation [5]:

$$
\begin{gathered}
\omega^{2}+2 \zeta \omega_{0} \omega+\omega_{0}^{2}=0 \\
\omega_{0}^{2} \equiv 1 / L C \\
\zeta \equiv \frac{1}{2} R /(L / C)^{1 / 2}
\end{gathered}
$$

where

$\omega$ actual resonant frequency

$\omega_{0}$ undamped resonant frequency

$\zeta$ damping factor

Though simple, the circuit gives us an opportunity to face up to the difficulties which are encountered with practical circuits and which are often ignored in the literature. We assume that the circuit has the following three features.

(1) The two output variables $\omega_{0}$ and $\zeta$ are statistically dependent. To meet the specifications their values must simulataneously fall within the limits

$$
\begin{gathered}
\omega_{0, \text { min }}<\omega_{0}<\omega_{0, \text { max }} \\
\zeta_{\min }<\zeta<\zeta_{\max }
\end{gathered}
$$

(2) The relationship between the three input variables 
TABLE I

THE PROBABILITY MASS FUNCTION FOR $\Phi_{C}\left(O_{C}\right)$

\begin{tabular}{lrr}
\hline$\Phi_{C}$ & \multicolumn{1}{c}{$O_{C}$} & $\mathrm{pmf}$ \\
\hline 0.80 & -0.0485 & 0.3 \\
1.0 & 0.0000 & 0.4 \\
1.2 & 0.0396 & 0.3 \\
\hline
\end{tabular}

and the two output variables is nonlinear as evidenced by (8).

(3) Two of the input variables $L$ and $R$ are statistically dependent. We shall return to this point later in connection with Tables 1 and 2.

To simplify the following discussion we normalize all variables with respect to their nominal values. The nominal values of $R, L, C, \omega_{0}$ and $\zeta$ are called $R_{\text {nom }}, L_{\text {nom }}, C_{\text {nom }}, \omega_{\text {nom }}$ and $\zeta_{\text {nom }}$.

The normalized input variables are $\Phi_{R}=R / R_{\text {nom }}, \Phi_{L}=$ $L / L_{\text {nom }}$, and $\Phi_{C}=C / C_{\text {nom }}$. The normalized output variables are $y_{0}=\omega_{0} / \omega_{0, \text { nom }}$ and $y_{\zeta}=\zeta / \zeta_{\text {nom }}$.

$$
\begin{aligned}
& y_{0}=\left(\Phi_{L} \cdot \Phi_{C}\right)^{-(1 / 2)} ; \\
& y_{\zeta}=\Phi_{R}\left(\Phi_{C} / \Phi_{L}\right)^{1 / 2} .
\end{aligned}
$$

In the general case, as here, we have after normalization a set of $m$ expressions for the $m$ normalized output variables, $y_{j}$, as functions of the $n$ input variables. The expressions will be of the following form:

$$
y_{j}=h_{j}\left(\Phi_{1}, \ldots, \Phi_{n}\right), \quad j=1, \ldots, m
$$

\subsection{The "Essentially Additive" Effect}

To use multidimensional convolution it frequently becomes necessary that we change the set of expressions of the form of (12) because we need expressions where in some form the effects of the input variables upon the output variables are additive. Equations (10) and (11) show that the output variables are determined by multiplication and division of the positive-valued input-variables. The obvious choice is the logarithmic transformation. It is monotonic, a feature which is desirable as it insures a one-to-one relationship between the values of the input-variable before and after transformation.

Use $z$ to denote the transformed output variables and $O$ to denote the transformed input variables. Then we have

$$
\begin{aligned}
z_{0} & \equiv \log y_{0}, \quad z_{\zeta} \equiv \log y_{\zeta} \\
O_{L} & \equiv \frac{1}{2} \log \Phi_{L}, \quad O_{C} \equiv \frac{1}{2} \log \Phi_{C}, \quad O_{R} \equiv \log \Phi_{R} ;
\end{aligned}
$$

all logs are to the base 10 .

Equations (10) and (11) become

$$
\begin{aligned}
& z_{0}=-O_{L}-O_{C} \\
& z_{\zeta}=O_{R}+O_{C}-O_{L}
\end{aligned}
$$

\begin{tabular}{|c|c|c|c|c|}
\hline$\Phi_{R}$ & $\Phi_{R} O_{L}$ & $\begin{array}{c}0.90 \\
-0.0229\end{array}$ & $\begin{array}{l}1.00 \\
0.0000\end{array}$ & $\begin{array}{l}1.10 \\
0.0207\end{array}$ \\
\hline 0.85 & -0.0706 & 0.20 & 0.05 & 0.05 \\
\hline 1.00 & -0.0000 & 0.05 & 0.30 & 0.05 \\
\hline 1.15 & 0.0607 & 0.05 & 0.05 & 0.20 \\
\hline
\end{tabular}

Equations (14) and (15) show that Assumption 1 is satisfied, since the change in $z_{0}$ and $z_{\zeta}$ due to changes in the values of $\Phi_{L}, \Phi_{C}$, and $\Phi_{R}$ are additive. This puts us in the fortunate
TABLE 2

\begin{tabular}{|c|c|c|c|c|c|c|}
\hline$\Phi_{C}$ & $\Phi_{L}$ & $\Phi_{R}$ & $\left(10^{4} z_{0}, 10^{4} z_{\zeta}\right)$ & $f_{C}$ & $f_{L R}$ & $g_{0 \zeta}=f_{C L R}$ \\
\hline 0.80 & 0.90 & 0.85 & $(714,-962)$ & 30 & 20 & $6^{*}$ \\
\hline 1.00 & 0.90 & 0.85 & $(229,-477)$ & 40 & 20 & 8 \\
\hline 1.20 & 0.90 & 0.85 & $(-167,-81)$ & 30 & 20 & 6 \\
\hline 0.80 & 1.00 & 0.85 & $(485,-1191)$ & 30 & 5 & $1.5^{*}$ \\
\hline 1.00 & 1.00 & 0.85 & $(0,-706)$ & 40 & 5 & 2 \\
\hline 1.20 & 1.00 & 0.85 & $(-396,-310)$ & 30 & 5 & 1.5 \\
\hline 0.80 & 1.10 & 0.85 & $(278,-1398)$ & 30 & 5 & $1.5^{*}$ \\
\hline 1.00 & 1.10 & 0.85 & $(-207,-913)$ & 40 & 5 & 2 \\
\hline 1.20 & 1.10 & 0.85 & $(-603,-517)$ & 30 & 5 & $1.5^{*}$ \\
\hline 0.80 & 0.90 & 1.00 & $(714,-256)$ & 30 & 5 & $1.5^{*}$ \\
\hline 1.00 & 0.90 & 1.00 & $(229,229)$ & 40 & 5 & 2 \\
\hline 1.20 & 0.90 & 1.00 & $(-167,625)$ & 30 & 5 & 1.5 \\
\hline 0.80 & 1.00 & $1.00^{\dagger}$ & $(485,-485)$ & 30 & 30 & 9 \\
\hline 1.00 & 1.00 & $1.00^{\dagger}$ & $(0,0)$ & 40 & 30 & 12 \\
\hline 1.20 & 1.00 & $1.00^{+}$ & $(-396,396)$ & 30 & 30 & 9 \\
\hline 0.80 & 1.10 & 1.00 & $(278,-692)$ & 30 & 5 & 1.5 \\
\hline 1.00 & 1.10 & 1.00 & $(-207,-207)$ & 40 & 5 & 2 \\
\hline 1.20 & 1.10 & 1.00 & $(-603,189)$ & 30 & 5 & $1.5^{*}$ \\
\hline 0.80 & 0.90 & 1.15 & $(714,351)$ & 30 & 5 & $1.5^{*}$ \\
\hline 1.00 & 0.90 & 1.15 & $(209,836)$ & 40 & 5 & 2 \\
\hline 1.20 & 0.90 & 1.15 & $(-167,1232)$ & 30 & 5 & $1.5^{*}$ \\
\hline 0.80 & 1.00 & 1.15 & $(485,122)$ & 30 & 5 & 1.5 \\
\hline 1.00 & 1.00 & 1.15 & $(0,607)$ & 40 & 5 & 2 \\
\hline 1.20 & 1.00 & 1.15 & $(-396,1003)$ & 30 & 5 & $1.5^{*}$ \\
\hline 0.80 & 1.10 & 1.15 & $(278,-85)$ & 30 & 20 & 6 \\
\hline 1.00 & 1.10 & 1.15 & $(-207,400)$ & 40 & 20 & 8 \\
\hline 1.20 & 1.10 & 1.15 & $(-603,796)$ & 30 & 20 & $6^{*}$ \\
\hline
\end{tabular}

THE BIVARIATF PROBABILITY MASS FUNCTION FOR $\Phi_{L}$ AND $\Phi_{R}\left(O_{L}\right.$ AND $\left.O_{R}\right)$

The body of the table gives the pmf.

TABLE 3

BIVARIATE PROBABILITY MASS FUNCTIONS FOR $\left(z_{0} z_{\zeta}\right)$

All $f$ and $g$ values are listed in percent. The * values fall outside the limits. The +illustrate the points mentioned in Section 6.1 .

position of having linear relationships between the input and output variables. The specified output intervals according to (13a); then become

$$
\begin{aligned}
& z_{0, \text { min }}<z_{0}<z_{0, \max } \\
& z_{\zeta, \min }<z_{\zeta}<z_{\zeta, \max }
\end{aligned}
$$

\subsection{Discrete Probability Mass Functions}

Tables 1 and 2 show the probability mass functions (pmf) for the input variables. Discrete variables have pmf's; continuous variables have pdf's. The variables are illustrated as being discrete, this quantization is solely to facilitate the later illustration in Table 3 and is not be confused with the quantization of $y_{i}$-values to be discussed in Section 6. Table 
2 shows the probabilistic relationship between $O_{L}$ and $O_{R}$; $\Phi_{C}$ and thereby $O_{C}$ is assumed to be statistically independent of the other two input-variables. $\Phi_{C}$ has the pmf illustrated in Table 2. Assumption 2 is justified because the trivariate pmf $f_{C L R}$ is the product of a bivariate $\operatorname{pmf} f_{L R}$ and the univariate $\operatorname{pmf} f_{C}$. The total number of combinations of the input variables is quite small, $3 \times 9=27$.

\subsection{The Transformed-Output-Parameter Plane}

We convolve the univariate pmf in Table 1 , with the bivariate pmf in Table 2 , and arrive at the 27 values of $\left(z_{0}, z_{\zeta}\right)$ in Table 3 ; with each value is associated a probability $f_{05}$ which clearly is the same as the probability $f_{C L R}$ of the corresponding set of input-parameters.

\subsection{The Reliability}

The nonasterisked points represent sets of input parameters which result in acceptable pairs of output-parameters as defined by (16) and (17) with the following limits : $z_{0, \min }=$ $-0.0500, z_{0, \text { max }}=+0.0500, z_{\zeta, \text { min }}=-0.1000, z_{\zeta, \text { max }}=$ +0.1000 . Ten points fall outside the rectangle. The probabilities of the 10 sets of input parameter values are indicated in Table 3 by asterisks ; their sum asterisked is $24 \%$. We have thus arrived at the answer to the question posed at the beginning of Section 5.1 : the reliability $\mathscr{R}$ is $100 \%-24 \%=$ $76 \%$.

\section{THE IMPORTANCE OF THE QUANTIZATION OPERATION}

\subsection{Why Quantization Is Necessary}

At this point the reader may rightly comment that we made the example simple by

(i) assuming that the univariate and the bivariate were discrete,

(ii) arranging things so that this dandy logarithmic transformation insured the "essentially additive effect" needed to satisfy Assumption 1.

Regarding (ii) we must admit that maybe it was too cute, but we could not resist the temptation to show by example what a timely transformation can do for a designer. Regarding (i) we are faced with a problem which can be alleviated by a suitable quantization of each output variable; this matter will now be discussed in detail.

We are concerned with the general case where Assumptions 1 and 2 are satisfied. Each of the $n \Phi_{i}$ has a set of limits and a pdf. Now keep all input variables at their nominal values except the first one. If we then let $\Phi_{i}$ ican the range from $\Phi_{i, \min }$ to $\Phi_{i, \max }$ each of the $y_{j}$ will trace a path in the $m$-dimensional output parameter space. If we restrict ourselves to the case $m=2$ our situation is in Fig. 1 where the continuous path illustrates the case with the continuous $\Phi_{i}$. If $\Phi_{i}$ is discrete we will instead of a continuous path get some discrete points. In the example there are 3 such points illustrating the three possible values of $\Phi_{C}$ when $\Phi_{L}=$ $\Phi_{R}=1$. The three points are readily determined from

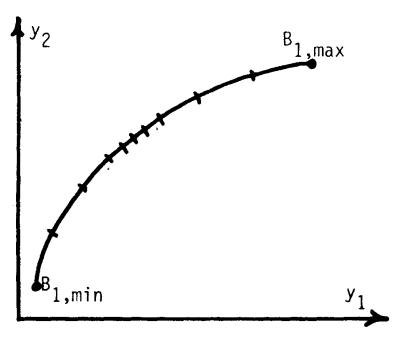

Fig. 1. The "line probability density." The boundaries (endpoints) are: $B_{1, \min }$ corresponds to $\Phi_{1}=\Phi_{1, \min } ; B_{1, \max }$ corresponds to $\Phi_{1}=\Phi_{1, \max }$.

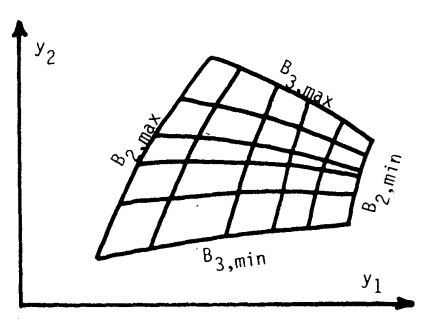

Fig. 2. The bivariate probability density. The boundaries are: $B_{2 . \min }$ corresponds to $\Phi_{2}=\Phi_{2, \min }, B_{2, \max }$ corresponds to $\Phi_{2}=\Phi_{2, \max } ; B_{3, \min }$ corresponds to $\Phi_{3}=\Phi_{3, \text { min }}, B_{3, \max }$ corresponds to $\Phi_{3}=\Phi_{3, \text { max }}$.

Table 3 ; they are shown by the ${ }^{\dagger}$.

We know from $f_{i}\left(\Phi_{i}\right)$ that some $\Phi_{i}$-values are more probable than others, i.e., certain sections of the path represent more likely values of $\left(y_{1}, y_{2}\right)$ than other sections. Symbolically, we have "spread one unit of probability mass" over the path according to how likely it is to find $\left(y_{1}, y_{2}\right)$ at each point. The changing likelihood is indicated in Fig. 1 by the nine short cross-bars on the path. The crossbars are so located that a $\left(y_{1}, y_{2}\right)$-value with $10 \%$ probability may be located in either of the 10 intervals. In the discrete case the display is simpler than in the continuous case because one can list the discrete probabilities at each point (as was done in Table 2).

In all the cases where the effect of $\Phi_{i}$ on $y_{j}$ can be illustrated by a 1 -argument function $h_{j, i}\left(\Phi_{i}\right)$, the resulting effect can be depicted as a path in output parameter space. If the effect of, say, $\Phi_{2}$ on $y_{j}$ depends on the true value of $\Phi_{3}$ our situation is more difficult; then the resulting effect is determined by a 2-argument function $h_{j, 23}\left(\Phi_{2}, \Phi_{3}\right)$, the function is analogous to the one in (4). We will now discuss the case with the 2-argument function in some detail.

As before we assume that both $\Phi_{2}$ and $\Phi_{3}$ each have a maximum and a minimum. We now let the point $\left(\Phi_{2}, \Phi_{3}\right)$ scan the allowed values while keeping all other input-variables at their nominal values, $\Phi_{i}=1, i=1,4,5, \ldots, n$. While doing so we scan a 2-dimensional subspace in the $m$-dimensional output-parameter space. In the case $m=2$ the subspace becomes the $\left(y_{1}, y_{2}\right)$-plane. The pdf function for the output variables $\left(y_{1}, y_{2}\right)$ is found by mapping $f_{23}\left(\Phi_{2}, \Phi_{3}\right)$ onto output parameter space. In Fig. 2 the varying closeness of the grid illustrates the changing bivariate $\left(y_{1}, y_{2}\right) \mathrm{pdf}$. The lines in the grid are so drawn that a $\left(y_{1}, y_{2}\right)$-value can be found with $4 \%$ probability in either of the " $5 \times 5=25$ " regions. 
Our goal is to determine the bivariate for $\left(y_{1}, y_{2}\right), g=$ $g\left(y_{1}, y_{2}\right)$. If all $n$ input variables are statistically independent and the effects of their variations upon $y_{1}$ and $y_{2}$ are additive (i.e., Assumptions 1 and 2 are satisfied) we can determine $g$ by convolving the $n$ "line probability densities" all of which look somewhat like the one in Fig. 1. Clearly the computations will be horrendous! If the statistical or functional relationship between two (or more) input variables must be taken into account their "line-densities" (like the one in Fig. 1) are replaced by a multivariate pdf (like the one in Fig. 2); this change clearly does not make the convolution any easier.

To overcome the computational difficulties just described we obviously somehow must change one or more constraints.

\subsection{The Quantization}

The constraint we will give up is that $y_{1}$ and $y_{2}$ are continuous functions. Actually what we are giving up is but a figment of our imagination: in engineering application the values of all variables are quantized simply because the number of decimals we use is limited. Instead of being a continuous variable we take $y_{j}$ to be a discrete variable with the values $k_{j} \cdot \Delta_{j} ; \Delta_{j}$ is a suitable constant and $k_{j}$ are integers, our running variables. For the 2-dimensional use, the $\left(y_{1}\right.$, $y_{2}$ ) pair of coordinate values now has been restricted to points on a 2-dimensional lattice in the plane.

Next we somehow must move the probability mass from the "line density" and the bivariate pdf to the lattice points. The relocation is carried out so that some suitable error measure is minimized. (The smaller the $\Delta_{1}{ }^{-}$and $\Delta_{2}$-values are, the smaller the quantization error will be.)

When all the probabilities, as illustrated in Figs. 1 and 2, have been relocated at lattice points it is a simple matter to perform the required number of 2-dimensional convolutions. The result of each convolution has the same format as the convolving functions: a number of discrete probabilities associated with specified lattice points. After each convolution, the number of lattice points with non-zero probability increases.

\section{AN APPLICATION OF THE MDCQ-METHOD}

The method was put to use in a recent study of circuitreliability vs. circuit-cost [6] where the reliabilities of 128 different implementations of a particular three-transistor amplifier were computed. As a check on the accuracy of the computed reliability-figures some of the reliabilities were recomputed using Monte Carlo techniques; in all cases there was good agreement.

\section{CONCLUSIONS}

In this paper we have presented a new and simple method called "Multi-dimensional Convolution with Quantization" by which we can solve the familiar problem [1] stated in the Introduction: Find the distribution of output parameters, given the distributions of the input parameters and the system topology.

The probability that all output parameters are simultaneously within their specifications is the reliability of the system.

To make the MDCQ-method work, two assumptions must be justified. They are milder than the assumptions of linearity and of statistical independence which are made so freely in the literature. The method introduces an error due to the quantization. The error may be made arbitrarily small by using smaller increments; the penalty is an increase in computation time. The method compares well to the other methods for solving the same problem.

\section{REFERENCES}

[1] M. L. Shooman, Probabilistic Reliability: An Engineering Approach. New York: McGraw-Hill, 1968, subsection 7.6.1.

[2] P. W. Becker and F. Jensen, Design of Systems and Circuits for Maximum Reliability and Maximum Production Yield. Section 5.5. To be published by Den Private Ingeniørfont, Copenhagen

[3] P. W. Becker and F. Jensen, op. cit., section 5.2.

[4] M. L. Shooman, op. cit., subsection 7.8.2.

[5] H. Chestnut and R. W. Mayer, Servomechanisms and Regulating System Design. New York: Wiley, 1959, vol. 1, equation 3.5-6.

[6] P. W. Becker and B. Jarkler, "A systematic procedure for the genertion of cost-minimized designs," IEEE Trans. Rel., vol. R-21, pp. 41-45, Feb. 1972.

Mailing address:

Peter W. Becker

Björn Jarkler

Electronics Laboratory

Technical University of Denmark

Bldg. 344

2800 Lyngby, Denmark

Peter W. Becker received his M.S. and dr. techn. degree in Electrical Engineering from the Technical University of Denmark. He is presently a member of the staff at the Electronics Laboratory of the Technical University of Denmark.

Dr. Becker's previous technical experience includes an eight year period with the General Electric Co., Electronics Laboratory, Syracuse, N.Y.

Dr. Becker has written several papers in the field of reliability. He is also interested in pattern recognition and published in 1971 the book, $A n$ Introduction to the Design of Pattern Devices, Springer-Verlag, Wien-New York: ISBN 3-211-81153-2. In 1974 he will publish the book Design of Systems and Circuits for Maximum Reliability or Maximum Production Yield (co-authored by Finn Jensen)

Björn Jarkler was born in Stockholm, Sweden in 1945. He received the M.S degree in electrical engineering from the Technical University of Denmark, Lyngby.

He has worked on electroacoustic equipment at the laboratory of Ortofon. Copenhagen. Denmark. He is alsn interested in medical electronics and is presently working on equipment for Danish hospitals. He is currently with the Electronics Laboratory, Technical University of Denmark. 